\title{
Resource Allocation and Reuse for Inter-Cell Interference Mitigation in OFDMA based Communication Networks
}

\author{
Zheng Xie and Bernhard Walke \\ Communication Networks (ComNets) Research Group \\ RWTH Aachen University \\ Aachen, Germany \\ \{tao|walke\}@comnets.rwth-aachen.de
}

\begin{abstract}
Inter-cell interference (ICI) mitigation is always a big challenge issue in cellular systems. In this work we propose an Enhanced Fractional Frequency Reuse (EFFR) scheme with an interference-aware reuse mechanism to achieve not only ICI limitation at cell edge but also enhancement of overall cell capacity in orthogonal frequency division multiple access (OFDMA) based communication networks. The EFFR scheme divides the whole available bandwidth into a Primary Segment and a Secondary Segment. The exclusive reuse-3 subchannels in the Primary Segment will be preferentially used by cell-edge users with higher transmission power, whereas the remaining subchannels are all reuse-1 subchannels allowing to be used with lower power. In addition, the resources in the Secondary Segment will be occupied by means of signal-to-interference-ratio (SINR) estimation. We implement the proposed EFFR scheme in a system-level simulator OpenWNS and compare its performance with the well-known Incremental Frequency Reuse (IFR) scheme, the classical reuse- 1 and reuse- 3 schemes. In order to reach a reliable evaluation, schemes are simulated with individual power masks, and using a scenario with surrounding cells up to $2^{\text {nd }}$-tier. The simulation results show that with the usage of the EFFR scheme substantial improvements in both, the overall cell capacity as well as the cell-edge user performance can be gained.
\end{abstract}

\section{INTRODUCTION}

The future wireless systems are envisaged to offer ubiquitous high date-rate coverage in large areas. Orthogonal frequency division multiple access (OFDMA) transmission technique is a promising candidate for that because OFDMA uses multi-channel OFDM approach and provides subcarrier access in the time and the frequency domain. Decisions to which timeslot, subchannel, and power level for communication are determined by the intelligent Medium Access Control (MAC) which seeks to maximize the signal-tointerference-ratio (SINR) for every Mobile Station (MS). OFDMA shows great benefits in handling inter-symbol interference, inter-carrier interference and supports high flexibility in the resource allocation. Even so, a big challenge issue with OFDMA still remained is co-channel interference or so-called inter-cell interference (ICI).

It is known that effective reuse of resources in a cellular system can highly enhance the system capacity. With a smaller frequency reuse factor (FRF), more available bandwidth can be obtained by each cell. So, in this sense the classical FRF of 1 is desirable. However, with the usage of FRF-1, the most MSs are seriously afflicted with heavy ICI, especially near the cell edge. And that causes severe connect outages and consequently low system capacity. The conventional method to figure out this problem is through increasing the cluster-order, which can mitigate the ICI efficiently, nevertheless at the cost of a decrease on available bandwidth for each cell. For a clusterorder 3 or 7 system, each cell is able to utilize just one third and one seventh of the total bandwidth respectively, which leads to restricted data transmission and lower system spectrum efficiency.

To take aim at improving cell-edge performance while retaining system spectrum efficiency of reuse-1, several solutions [1]-[5] have been proposed recently. Among them, the most representative approaches are the Soft Frequency Reuse (SFR) scheme [2], [4]-[5] and the Incremental Frequency Reuse (IFR) scheme [3]. These two methods concentrate on the high system spectrum efficiency with FRF-1 and efficient reduction of ICI (especially near the cell edge) simultaneously. However, both of them do not perform better than the classical reuse-1 scheme in full-load or overload situation. The overall cell capacity with the usage of the SFR scheme is even worse than the classical reuse-1 system with heavy-load traffic. Based on a deep analysis of these approaches, in this paper we will put forward a new design referred as Enhanced Fractional Frequency Reuse (EFFR) scheme for a better fulfillment of the goals, namely, to enhance the mean system capacity while restraining the ICI at cell edge. Moreover, as a solution with low system complexity and flexible spectrum usage is desirable, we will take systems with distributed radio resource management into account.

The remainder of this paper is organized as follows. In section II two well-known approaches SFR and IFR for cochannel interference mitigation in cellular OFDMA networks are outlined. Based on discussion of their advantages and drawbacks respectively, a novel Enhanced Fractional Frequency Reuse (EFFR) scheme is contributed in section III, which attempts to further improve the overall cell capacity while retaining a better cell-edge performance with the usage of FRF of 3 to the cell-edge users. Then, in section IV, simulation results of four different frequency schemes with 
different power masks are compared. Finally, the paper ends with some concluding remarks.

\section{SOFT FREQUENCY REUSE AND INCREMENTAL FREQUENCY REUSE}

\section{A. Soft Frequency Reuse Scheme}

The Soft Frequency Reuse (SFR) scheme, which has been adopted in the 3GPP-LTE system [1]-[2], addresses the challenge by increasing FRF and transmission power for celledge users, so that the ICI from contiguous cells to those users can be alleviated, and thereby to improve their performance.
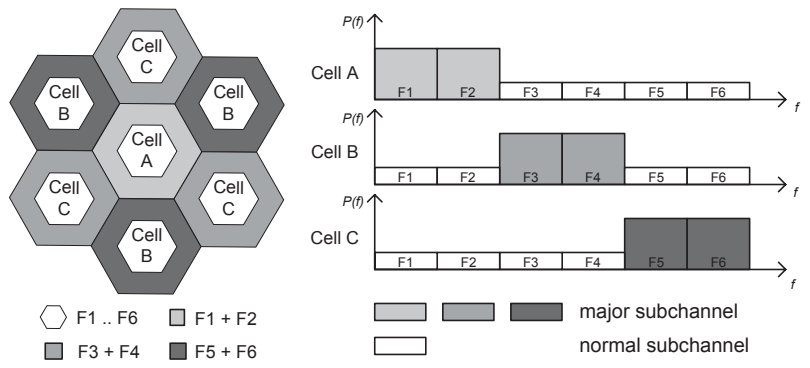

Fig. 1. Concept of the SFR scheme in a cellular system based on FRF $=3$ for the CEUs and FRF $=1$ for the CCUs.

The basic idea of the SFR scheme is applying FRF of 1 to cell-centre users (CCU) and FRF of 3 to cell-edge users (CEU) as illustrated in Fig. 1. Simply one third of the whole available bandwidth named Major Segment can be used by CEUs, yet on this Major Segment, packets are sent with higher power. To realize bigger FRF for CEUs, Major Segments among directly neighboring cells should be orthogonal. In opposite to the CEUs, the CCUs can access the entire frequency resources, however, with lower transmission power. A deeper insight to SFR can be found in [2].

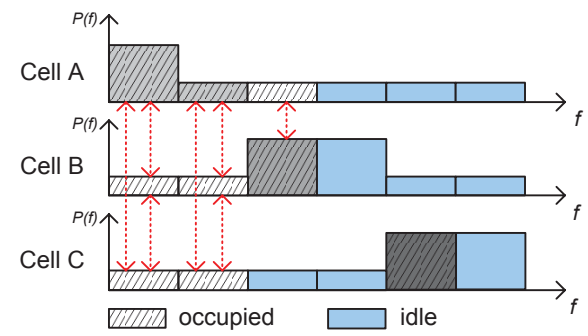

Fig. 2. More co-channel interferences even at low loading traffic situation with the usage of the SFR scheme.

For all that, some intrinsic limitations are exposed. For one thing, generally, there are more CEUs than CCUs in a cellular system, since the outer surface area is much larger than the inner part. However, with the SFR scheme CEUs have maximum one third of the entire bandwidth to utilize, which results in a lower spectrum efficiency. Next, as shown by a sketch in Fig. 2, more co-channel interferences could happen even in a low traffic load situation, while there are still subchannels in idle and underutilized in the system. This is because the resource allocation of all cells via the SFR scheme starts always from the first subchannel up. Lastly, in [3] Ki Tae
Kim et al. have given us a study result that with the usage of the SFR scheme the cell throughput is even inferior to the classical reuse-1 system when loading factor over 0.5. The reason is in a SFR system at most one third of the subchannels can be used to transmit data with higher power while die remaining two third subchannels work with lower power, which induces an overall throughput loss. In other words, the SFR ameliorates performance of the CEUs at the expense of degrading the overall cell capacity.

\section{B. Incremental Frequency Reuse Scheme}

Aiming at the limitations of the SFR scheme mentioned above, Ki Tae Kim et al. came up with a new design referred as Incremental Frequency Reuse (IFR) scheme, which can reduce the ICI effectively in the case of low offered traffic situation, and keeps the overall system capacity at the same time.

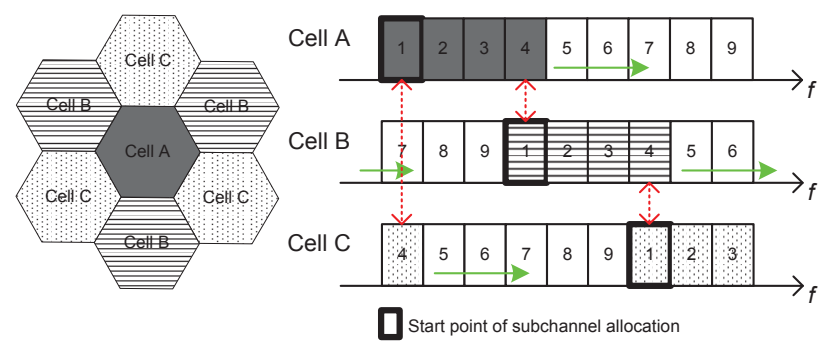

Fig. 3. Operation policy of the IFR scheme in a cellular system with 3 various types of neighboring cells.

The only difference between the IFR and the classical reuse-1 is, from which point of the available bandwidth it starts dispensing resources to the users. In an IFR system the directly adjoining cells assign resources from different subchannels. Fig. 3 exemplifies operation method of the IFR scheme for a cellular system with 3 various types of neighboring cells. Cells of type-A occupy resources from the first subchannel, whilst cells of type-B from one third of the whole bandwidth, and cells of type- $\mathrm{C}$ from two third of the bandwidth. They allocate consecutive subchannels successively along with traffic load increasing until the entire bandwidth is used up. A detailed description of the IFR scheme is presented in [3].

Resource assignment using the IFR scheme can overcome part of the limitations by applying the SFR scheme, namely the low spectrum reuse efficiency problem, the more co-channel interferences at low loading traffic problem and the loss of cell capacity system when system over half-full loaded. The ICI generated by directly adjoining cells can be avoided completely at low traffic situation, since frequency reuse of the first tier neighboring cells doesn't occur when loading factor below 0.3 , and the whole system works just like a classical reuse-3 system. In essence, by means of the IFR scheme, system operates with increasing traffic load like moving from a reuse-3 system to a reuse-1 system.

Although the most limitations of the SFR scheme can be eliminated by using the IFR scheme, it only performs better, when just fewer traffic exists in a system. When the loading factor is greater than 0.3 , though the IFR surpasses the classical reuse-1 scheme, it is inferior to the SFR scheme. With the help 
of its static configuration, the IFR scheme disperses the cochannel interferences, but with increasing traffics in the system, the CEUs are still interfered severely. Furthermore, the simulation results in [3] disclose another phenomenon that both schemes cannot perform better than the classical reuse-1 scheme in over-middle-load or full-load situations. The SFR scheme even performs worse than the reuse- 1 system. That is to say, the system capacity cannot be substantively improved by the IFR and the SFR schemes.

\section{ENHANCED Fractional FREQUENCY REUSE SCHEME}

The discussion about advantages and limitations of the both IFR and SFR schemes in the last section motivates us to propose a new design named Enhanced Fractional Frequency Reuse (EFFR) scheme, which attempts to retain the advantages of the both approaches while avoiding their limitations, and seeks to further enhance the system capacity especially in overload situations.

\section{A. Design Requirements}

The EFFR scheme is designed to meet the following requirements:

- Support flexibility with non-uniform user or traffic distribution

- Support adaptation to time varying traffic conditions

- Exploit possibility for self-setting up preferable reuse combinations

- No need for the resource coordination among different base stations (BS) in radio network controller (RNC) in the fixed resource allocation method

- Applicable for high FRF systems

- Low system complexity

\section{B. Concept of the Enhanced Fractional Frequency Reuse Scheme}

The objective of the proposed EFFR architecture is to improve system capacity while retaining better spectrum efficiency at cell edge. This can be achieved by basing on effectual mitigation of unwanted co-channel collisions for CEUs, maximizing the opportunities for the other users to choose suitable resources (time share and frequency share respectively) to reuse.

\section{1) Reuse Partition}

Just like the SFR scheme, the EFFR scheme defines 3 celltypes for directly neighboring cells in a cellular system, and reserves for each cell-type a part of the whole frequency band named Primary Segment, which is shown in the right part of Fig. 4 with thick border. The Primary Segments among different type cells should be orthogonal. Apart from the Primary Segment the remaining subchannels constitute the Secondary Segment. The Primary Segment of a cell-type is at the same time a part of the Secondary Segments belonging to the other two cell-types. Each cell can occupy all subchannels of its Primary Segment at will, whereas only a part of subchannels in the Secondary Segment can be used by this cell in interference-aware manner.

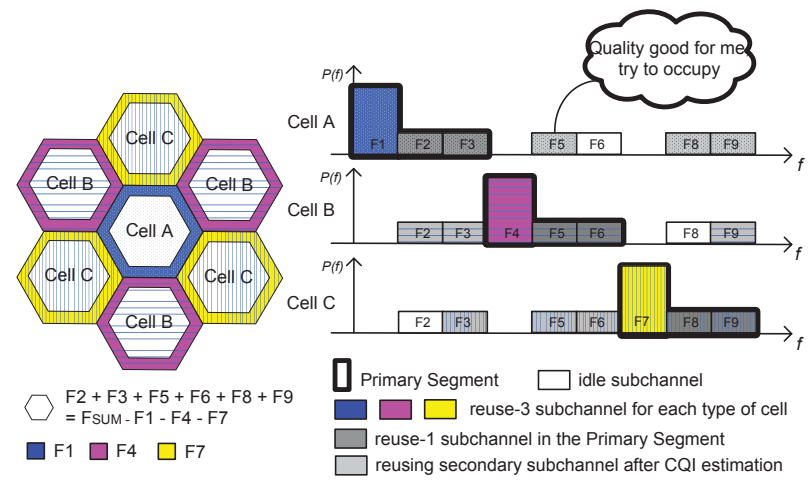

Fig. 4. Concept of the EFFR scheme in a cellular system based on partition of the exclusive reuse- 3 subchannels and reuse- 1 subchannels in the Primary Segment, as well as interference aware reuse on the Secondary Segment.

The Primary Segment of each cell will be further divided into a reuse- 3 part and reuse- 1 part. The reuse- 1 part can be reused by all types of cells, while reuse- 3 part can only exclusively be reused by other same type cells. The reuse- 3 subchannels cannot be reused by directly neighboring cells, that attenuates the co-channel interferences among them and therefore it is stipulated for the vulnerable CEUs to take priority of using these subchannels over CCUs.

\section{2) Power Loading}

The total transmission power is kept constant for all reuse schemes depicted in this paper. Since any cell-type (e.g. celltype-A in Fig. 4) in the EFFR scheme cannot use the reuse-3 subchannels dedicated to the other two cell-types (e.g. celltype-B and $-\mathrm{C}$ in Fig. 4) within their Primary Segments, the power on its reuse-3 subchannels can be tripled without decreasing the power for other available reuse-1 subchannels.

\section{3) SINR Estimation}

Since a cell acts on the Secondary Segment as a guest, and occupying secondary subchannels is actually reuse the primary subchannels belonging to the directly neighboring cells, therefore reuse on the Secondary Segment by each cell should conform to two rules:

- $\quad$ monitor before use and

- resource reuse based on SINR estimation.

Each cell listens on every secondary subchannel all the time. And before occupation, it makes SINR evaluation according to the gathered channel quality information (CQI) and chooses resources with best estimation value for reuse. If all available secondary resources are either occupied or not good enough to a link, it will give up reusing for this link. This will not lead to resource wasting, which means some resources maybe not reusable for this link, but can be reused by other links. And another thereby gained merit is that it will not generate excessive interference for the neighboring cells which would degrade their performance. So, an upgrade of spectrum efficiency is expected by using the interference-aware-reuse mechanism on the Secondary Segment. 


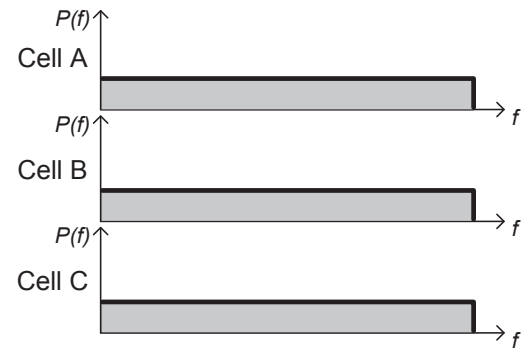

(a) Classical reuse-1 \& IFR

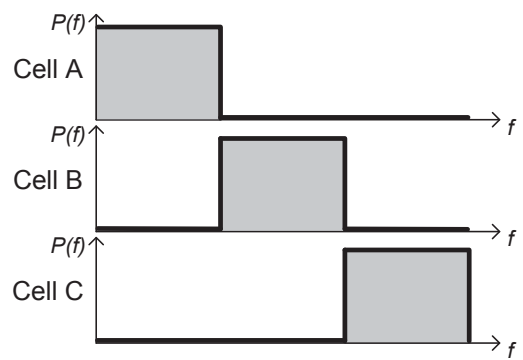

(b) Classical reuse-3

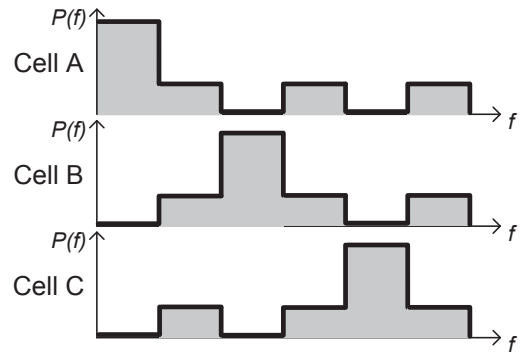

(c) EFFR

Fig. 5. Cell-specific power masks over system bandwidth for classical reuse-1, IFR, reuse-3 and EFFR.

On the other hand, all above elucidation is based on a precise SINR estimation. However, an improper modulation and coding scheme (PHY-mode) selection due to a bad SINR estimation would cause to either higher packet loss rate or lower spectral efficiency, and thereupon wastes precious resources. Hence, to have a reliable SINR estimation is a crucial factor for maximizing system spectrum efficiency.

4) Resource Allocation

The details of the EFFR operation principles are as follows:

1. The reuse-3 subchannels will be assigned to CEUs with the usage of the proportional faire scheduling strategy. If there are still resources remained after all CEUs are served, they will be continuing allotted to CCUs who can only transmit packets utilizing relatively worse PHY-mode.

2. When the reuse-3 subchannels are exhausted, the remaining reuse- 1 subchannels in the Primary Segment are allocated to residual unsatisfied users using maximum throughput strategy until demands of all users are met or the entire Primary Segment is occupied.

3. If still resources are requested, available reuse-1 subchannels in the Secondary Segment will be scheduled to adequate users by applying interferenceaware- operation.

\section{Distinctions between the EFFR and the other two afore- mentioned Schemes}

The EFFR scheme owns mainly the following salient features, which are typically different to the SFR and IFR schemes:

- $\quad$ Since the users at cell edge are very weak at resisting co-channel interferences, the reuse-3 subchannels in the Primary Segment for each cell are exclusively available for the users in the same type cell. This means real reuse- 3 is applied on these subchannels, and for each cell not the whole bandwidth is available.

- In order to advance spectral efficiency, users which are allotted shares of the reuse-3 subchannels, should send packets with higher transmission power, whether they are CCUs or CEUs. In contrast, to reduce excessive interferences to the neighboring cells and avoid unwanted power wasting, packets will be sent on a reuse-1 subchannel in lower strength.

- Allocation of reuse-1 subchannels in the Secondary Segment is not blindly carried out, but in an interference-aware way according to the SINR estimation.

- In the Primary Segment unsatisfied users, whether they are CCUs or CEUs, have the same chance to get resources in the Secondary Segment, if it can find usable resource in accordance with the SINR estimation.

Besides the basic design of the EFFR scheme, there are some relevant parameters which play paramount roles in the realization and could influence the system performance severely, such as: 1) the ratio of the number of reuse-3 subchannels $\boldsymbol{M}$ to reuse-1 subchannels $\boldsymbol{N}$ in the Primary Segment; 2) the power ratio employed on reuse-3 subchannels to reuse-1 subchannels; 3) range definition for partition of CCUs and CEUs; 4) SINR threshold for reuse etc.. In what follows, we will focus on the affect on performance of using the EFFR scheme with varying ratios of $\boldsymbol{M}$ to $\boldsymbol{N}$ in the Primary Segment.

\section{EVAluation}

The Open Wireless Network Simulator (OpenWNS) [8] is a framework for the implementation of event driven wireless network protocol simulators. It has been developed at the Chair of Communication Networks RWTH Aachen University, and is used for the implementation of several wireless network protocols like GSM, UMTS, IEEE 802.11, IEEE 802.16 [6]. We integrated the IFR and proposed EFFR schemes into the so-called WiMAC module, which is an implementation of the IEEE 802.16 standard in the OpenWNS.

With simulations we aim to demonstrate the effectiveness of the proposed EFFR scheme in terms of improvement of CEU throughput as well as mean cell capacity. In respect that in most traffic load situations, the SFR scheme performs inferior to the IFR scheme which is exposed in [3], we will not discuss the SFR scheme in the succeeding part of this paper any more, but we will compare the devised EFFR scheme with the classical reuse-1, reuse-3 and the IFR schemes. Furthermore, the EFFR scheme with three $\boldsymbol{M}$ to $\boldsymbol{N}$ combinations (8:2|7:3|6:4) are evaluated. 
An OFDMA uplink cellular system in an omni-cell case for simulations is considered. User terminals are uniformly distributed within each hexagonal cell. We assume the total system transmission power is kept constant, and each user terminal has a maximal transmission power of $200 \mathrm{~mW}$. Therefore we use different cell-specific power masks in response to diverse spectrum usage for all studied approaches as given in Fig. 5. A power mask prescribes transmit power a user uses depending on the part of the spectrum. Since for the classical reuse- 1 scheme and the IFR scheme users of all cells can use the whole system bandwidth, the total system transmission power is thereby evenly distributed over the whole bandwidth (Fig. 5a), whereas for the reuse-3 scheme the power masks block all but one third of the spectrum (Fig. 5b). A corresponding power masks example for the proposed EFFR scheme is illustrated in Fig. 5c (also displayed in Fig. 4b). The main relevant parameters used in simulations are shown in Table I. And switching thresholds for the PHY-modes in [7] was adopted.

TABLE I

SIMULATION PARAMETERS

\begin{tabular}{|c|c|}
\hline Parameter & Value \\
\hline System bandwidth & $20 \mathrm{MHz}$ \\
\hline Center frequency & $5470 \mathrm{MHz}$ \\
\hline Subcarriers (FFT size) & 2048 \\
\hline OFDMA symbol duration & $102.858 \mu \mathrm{s}$ \\
\hline Number of subchannels & 30 \\
\hline Frame length & $10 \mathrm{~ms}$ \\
\hline DL-subframe : UL-subframe & $1: 1$ \\
\hline UT transmission power & $23 \mathrm{dBm}$ \\
\hline Noise figure at [BS, MS] & {$[5,7] \mathrm{dB}$} \\
\hline Cell radius & $1100 \mathrm{~m}$ \\
\hline Range for CEUs & $500 \mathrm{~m} \sim 1100 \mathrm{~m}$ \\
\hline Number of interfering cells & 18 (up to $2^{\text {nd }}$ tier) \\
\hline Path loss exponent & 2.9 \\
\hline UT thermal noise density & $-174 \mathrm{dBm} / \mathrm{Hz}$ \\
\hline Traffic model & symmetric, neg. exp IAT \\
\hline Offered traffic for each user & $333 \mathrm{kbit} / \mathrm{s}$ \\
\hline
\end{tabular}

Fig. 6 shows the average cell throughput as a function of the number of users in each cell. From the results, we can see that the EFFR scheme outperforms the other three schemes almost in every situation regardless of which $\boldsymbol{M}$ to $\boldsymbol{N}$ combination. The EFFR scheme has a close performance to the classical reuse-3 scheme, but is much better than the IFR and the classical reuse-1 schemes, when the number of users increases till 10. This implies, although the available bandwidth for the classical reuse-3 scheme is one third of the total bandwidth, the gain in SINR can counteract the loss in the bandwidth.

With increasing the number of users, the advantages of EFFR become significant. The EFFR provides better overall cell capacity than all the other schemes. With 18 users in the system, the EFFR scheme with $\boldsymbol{M}$ to $\boldsymbol{N}=8: 2$ combination reaches its peak rate around $2.8 \mathrm{Mbit} / \mathrm{s}$, whereas the other schemes also reach their peak rate of about $2.3 \mathrm{Mbit} / \mathrm{s}$ for the classical reuse- 3 scheme, $1.4 \mathrm{Mbit} / \mathrm{s}$ for the reuse- 1 scheme and 1.2 Mbit/s for the IFR scheme respectively. That discloses a fact that the EFFR scheme achieves an considerable increase of nearly $20 \%$ compared to the classical reuse- 3 scheme, and even has more than $100 \%$ gain over the classical reuse- 1 and the IFR scheme.

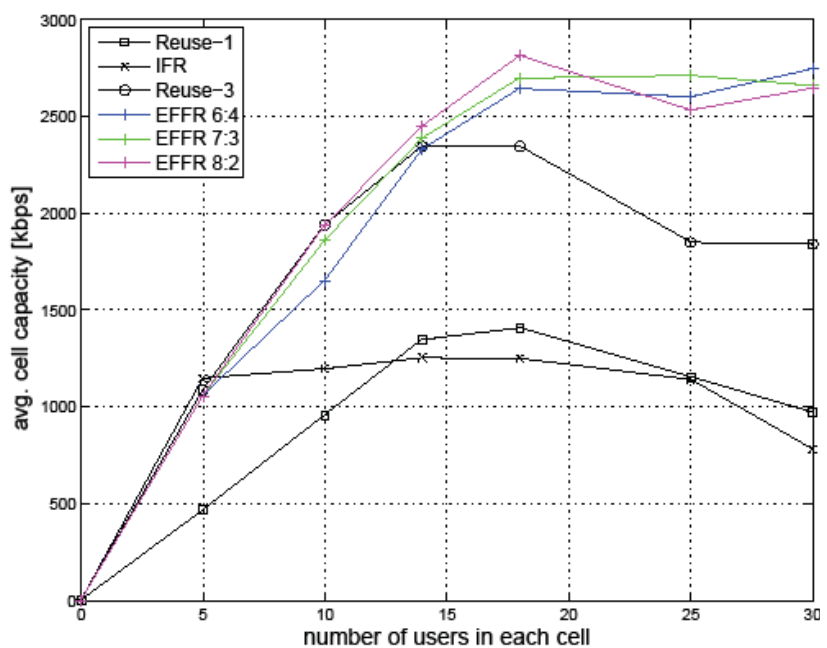

Fig. 6. Mean overall uplink cell capacity of four frequency reuse schemes as a function of the number of users in each cell, assuming users uniformly distributed over all cells in the system.

After this point, with a further user-number increasing, the performance of the classical reuse-3 scheme deteriorates quickly, whereas the EFFR keeps its cell throughput relatively stable in the vicinity of $2.6 \mathrm{Mbit} / \mathrm{s}$. As a consequence, the EFFR is able to obtain $40 \%$ gain over the conventional reuse- 3 scheme in the end, which coincides with the fact that the proposed EFFR scheme has $40 \%$ more available bandwidth (14 subchannels) than the classical reuse-3 scheme (10 subchannels).

Nevertheless, when we make a scrutiny into the curves, we might notice that the mean system throughput of the EFFR scheme with $\boldsymbol{M}$ to $\boldsymbol{N}$ combinations of $7: 3$ and $6: 4$ are slightly worse than that of the reuse- 3 scheme, when the number of users is around 10. This is mainly because there are CEUs which can only perform well using reuse-3 subchannels in the Primary Segment with higher power (M: 7 or 6), but they are strongly interfered by using reuse- 1 subchannels with lower power ( $N: 3$ or 4$)$. And in the case of 10 users, it is obviously that too much secondary reuse- 1 resources are reserved, which might be unavailable for these CEUs pursuant to the SINR estimation. This leads to a lower mean system throughput than which using the classical reuse- 3 scheme.

The detailed observations in terms of a CEU and a CCU performance can be found in Fig. 7. A clear advantage with the usage of researched EFFR scheme for each CCU is revealed in Fig. 7a. And Fig. 7b shows that a CEU by using the EFFR can get a close performance to that by using the classical reuse-3 scheme, and performs significantly better than the IFR and the classical reuse-1 schemes. From both figures together, we can arrive at such a conclusion that based on ensuring the performance of the CEUs close to the performance with the classical reuse- 3 scheme, the EFFR promotes the performance 
of the CCUs by peeling off part resources from the reuse-3 resources to lunch into the reuse-1 utilization.

As a consequence, the average cell throughput is enhanced due to the increase of available bandwidth while retaining lower inferences at the cell edge. The both Fig. 6 and Fig. 7 expose that an EFFR scheme with $\boldsymbol{M}$ to $\boldsymbol{N}=8: 2$ is the best combination among all.

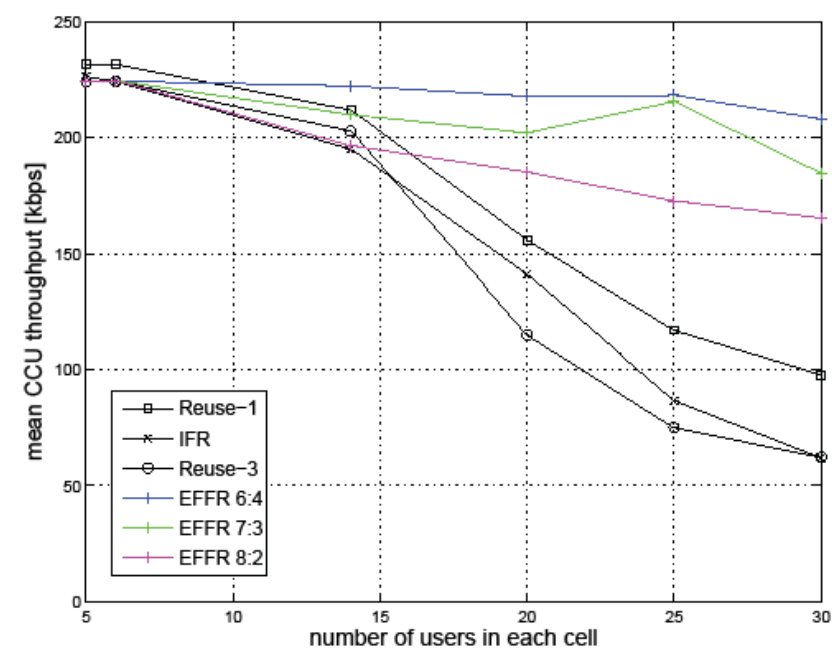

(a) Mean uplink throughput of CCU

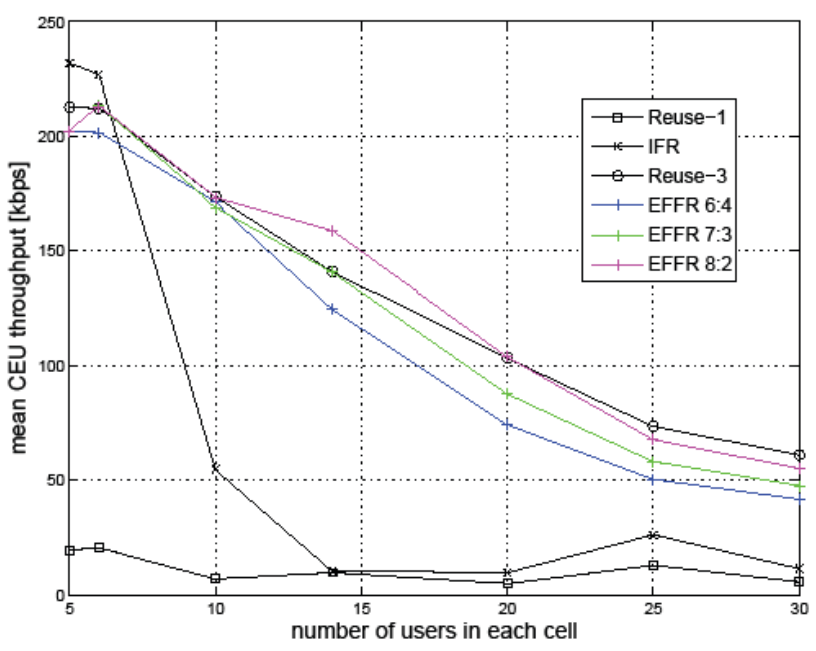

(b) Mean uplink throughput of CEU

Fig. 7. Mean uplink throughput of each user as a function of the number of users in each cell, having the same environment as in Fig. 6.

\section{CONCLUSION}

In this paper an enhanced frequency reuse scheme, the EFFR scheme, for co-channel interference mitigation in OFDMA networks is developed and evaluated. It designs a resource allocation and reuse mechanism and can provide a noteworthy improvement with the help of the CQI estimation. In terms of the inherent vulnerability of CEUs, the EFFR scheme reserves resources for them with two emphasizes: 1) using dedicated FRF-3; 2) with higher transmission power. Taking advantage of the geographic predominance of CCUs, the EFFR scheme allows them to occupy resources with FRF-1 and lower power. A detailed performance evaluation by means of event driven stochastic simulations is presented, whereby the EFFR scheme is compared with the conventional reuse-1 scheme, the reuse-3 scheme and the IFR scheme, which is proved outperforming the well-known SFR scheme adopted in LTE. The presented results show significant capacity gains and increases at cell edge achievable with the deployment of the proposed EFFR scheme. In conclusion, with the usage of the EFFR scheme the medium is able to be more effectively utilized, and the performance of all users including both CEUs and CCUs can be advanced.

\section{REFERENCES}

[1] 3GPP; Technical Specification Group Radio Access Network, "Physical channels and modulation (release 8)," TS-36.211, Jun. 2007, version 1.2.0.

[2] 3GPP; Huawei, "Soft frequency reuse scheme for UTRAN LTE", R1050507, May 2005.

[3] K. T. Kim, S. K. Oh, "An Incremental Frequency Reuse Scheme for an OFDMA Cellular System and Its Performance," in Proc. of the $67^{\text {th }}$ IEEE Vehicular Technology Conference (VTC-Spring 08), May 2008.

[4] M. Bohge, J. Gross, A. Wolisz, "Optimal Power Masking in Soft Frequency Reuse based OFDMA Networks," in Proc. of the $15^{\text {th }}$ European Wireless Conference (EW2009), pp. 162-166, Aalborg, Denmark, May 2009.

[5] Y. Xiang, J. Luo, "Inter-cell interference mitigation through flexible resource reuse in OFDMA based communication networks," in Proc. of the $13^{\text {th }}$ European Wireless Conference (EW2007), pp. 1-7, Paris, France, April 2007.

[6] IEEE 802.16-2004, “ IEEE standard for local and metropolitan area networks-Part 16: Air interface for fixed broadband wireless access systems," Oct. 1, 2004

[7] C. Hoymann, "Analysis and performance evaluation of the OFDMbased metropolitan area network IEEE 802.16," in Computer Networks, Volume 49 (2005) 341-363.

[8] D. Bültmann, M. Mühleisen, K. Klagges, M. Schinnenburg, "OpenWNS - open Wireless Network Simulator," in Proc. of the 15th European Wireless Conference 2009, pp. 205-210, Aalborg, Denmark, May 2009. 